\title{
CORRECTIONS AND EXTENSIONS OF “OPTIMAL CONTROL OF LINEAR SYSTEMS WITH ALMOST PERIODIC INPUT" BY G. DA PRATO AND A. ICHIKAWA*
}

\author{
BIRGIT JACOB ${ }^{\dagger}$, MIKAEL LARSEN ${ }^{\ddagger}$, AND HANS ZWART ${ }^{\S}$
}

\begin{abstract}
G. Da Prato and A. Ichikawa consider in their 1987 paper the optimal control problem for linear systems with almost periodic inputs. In order to get their results they use the space $B_{a p}^{2}(H)$, the completion of the space of almost periodic functions in the quadratic mean. They used that $B_{a p}^{2}(U)$-inputs give almost periodic outputs. By means of a scalar example we show that this does not hold in general. We prove that one of their main results still holds. Furthermore, we extend this result to all forcing terms in $B_{a p}^{2}(H)$ and show that if the forcing term is almost periodic, then the optimal control is almost periodic as well.
\end{abstract}

Key words. almost periodic functions, optimal control problem, tracking problem

AMS subject classifications. 49J15, 93C25, 42A75, 42A10

PII. S0363012996304614

1. Preliminaries and counterexample. In this paper we study the quadratic optimal control problem for infinite-dimensional linear systems with a forcing term. The forcing term will lie in the Besicovitch space of almost periodic functions. For examples of optimal control problems with this class of forcing terms we refer to [8] and [9]. It will be shown that for forcing terms in the Besicovitch class of almost periodic functions the optimal control problem has a unique solution which is again in this space. In section 2 we will solve the quadratic optimal control problem, and in this section we discuss some preliminary results concerning the Besicovitch class of almost periodic functions.

We begin by introducing some notation and stating some results concerning almost periodic functions. For more details we refer the reader to [1], [2], [3], [10]. Although the results in these references deal mostly with scalar almost periodic functions, the extension to the vector-valued case is straightforward.

Let $H$ be a separable Hilbert space. By $T P(H)$ we denote the space of trigonometric polynomials with values in $H$, i.e., $f \in T P(H)$ if and only if

$$
f(t)=\sum_{k=1}^{K} a_{k} e^{i \lambda_{k} t} \text { with } \quad a_{k} \in H, \lambda_{k} \in \mathbb{R}, K \in \mathbb{N} .
$$

On the space $T P(H)$ we can define the norm

$$
\|f\|_{\infty}:=\sup _{t \in \mathbb{R}}\|f(t)\|_{H} .
$$

If we take the completion with respect to this norm, then we get the space of almost periodic functions $A P(H)$; see [1] or [2]. However, we can define other norms on

*Received by the editors May 31, 1996; accepted for publication (in revised form) June 10, 1997; published electronically May 28, 1998.

http://www.siam.org/journals/sicon/36-4/30461.html

$\dagger$ Institut für Mathematik, Universität Dortmund, D-44221 Dortmund, Germany (jacob@math. uni-dortmund.de).

¥Mathematical Institute, Technical University of Denmark, Building 303, 2800 Lyngby, Denmark.

$\S$ Department of Applied Mathematics, University of Twente, P. O. Box 217, 7500 AE Enschede, the Netherlands (twhans@math.utwente.nl). 
$T P(H)$. For instance,

$$
\|f\|_{2}:=\sqrt{\lim _{T \rightarrow \infty} \frac{1}{2 T} \int_{-T}^{T}\|f(t)\|_{H}^{2} d t .}
$$

It is easy to see that this norm is associated with the inner product

$$
\langle f, g\rangle_{2}:=\lim _{T \rightarrow \infty} \frac{1}{2 T} \int_{-T}^{T}\langle f(t), g(t)\rangle_{H} d t .
$$

Taking the completion of $T P(H)$ with respect to the norm (1.3) gives the Besicovitch space of almost periodic functions $B_{a p}^{2}(H)$; see [2, Chapter II, sections 2 and 7]. This is a nonseparable Hilbert space; see [10, pp. 108 and 121]. Furthermore, we have that $[2$, pp. 73 and 76$]$

$$
A P(H) \hookrightarrow B_{a p}^{2}(H) .
$$

For every function in $T P(H)$ we can define the Bohr-transform as

$$
a(\lambda, f):=\lim _{T \rightarrow \infty} \frac{1}{2 T} \int_{-T}^{T} f(t) e^{-i \lambda t} d t .
$$

It is not hard to see that this can be continuously extended to the space $B_{a p}^{2}(H)$. Furthermore, this transformation gives an isometric isomorphism between $B_{a p}^{2}(H)$ and $\ell^{2}(\mathbb{R} ; H)$; see $\left[10\right.$, p. 122]. Using this, we identify an element $f \in B_{a p}^{2}(H)$ with its corresponding Fourier coefficients $a(\cdot, f)$. Since for an element $a(\cdot) \in \ell^{2}(\mathbb{R} ; H)$, we have that $a(\lambda)=0$ except for a countable number of $\lambda$ 's, we can write

$$
f(t) \sim \sum_{k \in \mathbb{N}} a\left(\lambda_{k}, f\right) e^{i \lambda_{k} t} .
$$

This we call the Fourier series of $f$.

Since we have constructed $B_{a p}^{2}(H)$ as the completion of the trigonometric polynomials, it is not immediately clear how this space is related to the class of Lebesgue measurable functions. From [10, pp. 102-108], it follows that in every equivalence class of $B_{a p}^{2}(H)$ there is a function that is locally square integrable. However, this does not imply that the Fourier series (1.5) converges in a classical sense, and we see (1.5) as a formal expansion that does not need to have a meaning pointwise in $t$, nor almost everywhere in $t$. This remark is illustrated in Example 1.3.

Now we shall give a meaning to the convolution mapping on $B_{a p}^{2}(H)$. The convolution operator associated with the exponentially stable $C_{0}$-semigroup $T(t)$ will be denoted by $\Phi: T P(H) \rightarrow T P(H)$ and is given by

$$
(\Phi f)(t):=\int_{-\infty}^{t} T(t-\tau) f(\tau) d \tau, \quad f \in T P(H) .
$$

It is easy to see that $\Phi$ is linear and that for $z \in H$ and $\lambda \in \mathbb{R}$, we have

$$
\left(\Phi\left[z e^{i \lambda \cdot}\right]\right)(t)=(i \lambda I-A)^{-1} z e^{i \lambda t} .
$$

Since the trigonometric polynomials are dense in $B_{a p}^{2}(H)$, the following lemma follows easily. 
LEMma 1.1. The operator $\Phi$ as defined in (1.6) has a unique extension in $\mathcal{L}\left(B_{a p}^{2}(H)\right)$ (again denoted by $\left.\Phi\right)$. Furthermore, for $f \in B_{a p}^{2}(H)$ with the Fourier series

$$
f(t) \sim \sum_{k=1}^{\infty} a_{k} e^{i \lambda_{k} t}
$$

$\Phi f$ has the Fourier series

$$
(\Phi f)(t) \sim \sum_{k=1}^{\infty}\left(i \lambda_{k} I-A\right)^{-1} a_{k} e^{i \lambda_{k} t},
$$

where $A$ is the infinitesimal generator of $T(t)$. The norm of $\Phi$ equals $\sup _{\lambda \in \mathbb{R}} \|(i \lambda I-$ $A)^{-1} \|$.

REMARK 1.2. The operator $\Phi$ also has a unique extension in $\mathcal{L}(A P(H)$ ) (again denoted by $\Phi)$ and its operator norm is bounded by $\int_{0}^{\infty}\|T(t)\| d t$.

In [9] it was claimed that the operator $\Phi$ maps $B_{a p}^{2}(H)$ into $A P(H)$. The following example shows that this does not hold in general.

EXAMPLE 1.3. Consider the exponentially stable semigroup $T(t)=e^{-t}$ and take the function $f \in B_{a p}^{2}(\mathbb{C})$ defined as

$$
f(t) \sim \sum_{n=1}^{\infty} \frac{1}{k\left(1-\frac{i}{\pi^{k}}\right)} e^{\frac{i}{\pi^{k}} t} .
$$

We calculate

$$
(\Phi f)(t) \sim \sum_{k=1}^{\infty} \frac{1}{k\left(1+\pi^{-2 k}\right)} e^{\frac{i}{\pi^{k}} t}
$$

and the idea is to show that $\Phi f \notin A P(\mathbb{C})$, using [6, Theorem 1.25]. In that theorem it is stated that if the exponents, $\lambda_{k}$, of an almost periodic function are linearly independent, then the Fourier series associated with $f(t)$ is uniformly convergent. The exponents $\left\{\lambda_{k}\right\}$ are said to be linearly independent if for any finite subset the relation

$$
\sum_{k=1}^{n} r_{k} \lambda_{k}=0
$$

with $\left\{r_{k}\right\}_{k=1 \ldots n}$ rational numbers implies that $r_{1}=\cdots=r_{n}=0$. The existence of a nonzero set of coefficients for our set of exponents would imply that $\pi$ is the root of a polynomial with integer coefficients, i.e., that $\pi$ is an algebraic number. It is known (see, e.g., [4]) that this is not the case, whence the exponents are linearly independent. However, for $t=0$ we get the sum

$$
\sum_{k=1}^{\infty} \frac{1}{k\left(1+\pi^{-2 k}\right)}
$$

which does not converge, and the Fourier series is not uniformly convergent. Therefore, we conclude that $\Phi f \notin A P(\mathbb{C})$. 
2. Linear optimal control problem. In this section we will study the linear optimal control problem for systems with forcing terms in $B_{a p}^{2}(H)$. We consider the system

$$
\dot{x}(t)=A x(t)+B u(t)+f(t),
$$

where $A: D(A) \rightarrow H$ is the generator of a $C_{0}$-semigroup $T(t), B \in \mathcal{L}(U, H)$, and $f \in B_{a p}^{2}(H)$. The pair $(A, B)$ is assumed to be stabilizable and we suppose that the input $u$ is in $B_{a p}^{2}(U)$.

Until now it has not been clear how to define a solution of (2.1). The problem is that in general $x(t)$ will be only an element of $B_{a p}^{2}(H)$, and thus it is not possible to consider initial states like $x(0)=x_{0}$. To overcome this difficulty, we consider so-called initially-at-rest solutions.

Definition 2.1. Let $f \in B_{a p}^{2}(H)$ and $u \in B_{a p}^{2}(U)$. Then $B u+f$ has a unique representation as

$$
[B u+f](t) \sim \sum_{k=1}^{\infty} y_{k} e^{i \lambda_{k} t}, \quad \lambda_{k} \in \mathbb{R}, \quad y_{k} \in H .
$$

We call the input $u$ an admissible input $\left(u \in U_{a d}(f)\right)$ if and only if there exists a sequence $\left(x_{k}\right)_{k} \subset D(A)$ such that $\left(\left\|x_{k}\right\|\right)_{k} \in \ell_{2}$ and $\left(i \lambda_{k} I-A\right) x_{k}=y_{k}$ for every $k \in \mathbb{N}$.

An initially-at-rest solution of (2.1) corresponding to the input $u$ and the forcing term $f$ is given by

$$
x(u, f)(t) \sim \sum_{k=1}^{\infty} x_{k} e^{i \lambda_{k} t}
$$

By definition, $x(u, f)$ lies in $B_{a p}^{2}(H)$.

In general, if the pair $(A, B)$ is merely stabilizable, there can be spectral points on the imaginary axis. Thus for one admissible input $u \in U_{a d}(f), f \in B_{a p}^{2}(H)$, there possibly exist more than one initially-at-rest solution of (2.1). If $i \mathbb{R} \subset \rho(A)$, then for every admissible input there exists a unique initially-at-rest solution, and this initially-at-rest solution is given by

$$
x(u, f)(t) \sim \sum_{k=1}^{\infty}\left(i \lambda_{k} I-A\right)^{-1} y_{k} e^{i \lambda_{k} t} .
$$

For instance, $i \mathbb{R} \subset \rho(A)$ is satisfied if $A$ generates an exponential stable $C_{0}$-semigroup $T(t)$. Moreover, exponential stability of $A$ implies $U_{a d}(f)=B_{a p}^{2}(U)$ for every $f \in$ $B_{a p}^{2}(H)$; i.e., in this situation every input in $B_{a p}^{2}(U)$ is admissible.

The term "initially-at-rest solution" was used in [7, p. 175], and it is motivated from the following observation. If the $C_{0}$-semigroup $T(t)$ is exponentially stable, $f \in T P(H)$ and $u \in T P(U)$, then the initially-at-rest solution of (2.1) can be written as

$$
x(u, f)(t)=\int_{-\infty}^{t} T(t-\tau)[B u(\tau)+f(\tau)] d \tau,
$$

where no initial condition is specified.

In the special situation where $u$ and $f$ are trigonometric polynomials, it is not hard to show that all initially-at-rest solutions are classical solutions. 
Since the pair $(A, B)$ is stabilizable, there exists an operator $F \in \mathcal{L}(H, U)$ such that $A-B F$ generates an exponentially stable $C_{0}$-semigroup $T_{-B F}(t)$. The following proposition shows that $U_{a d}(f) \neq \emptyset$ for every $f \in B_{a p}^{2}(H)$.

Proposition 2.2. Let $f \in B_{a p}^{2}(H)$ and $v \in B_{a p}^{2}(U)$ with Fourier series

$$
[B v+f](t) \sim \sum_{k=1}^{\infty} z_{k} e^{i \lambda_{k} t}, \quad z_{k} \in H, \quad \lambda_{k} \in \mathbb{R} .
$$

Defining $\tilde{x} \in B_{a p}^{2}(H)$ by

$$
\tilde{x}(t) \sim \sum_{k=1}^{\infty}\left(i \lambda_{k} I-A+B F\right)^{-1} z_{k} e^{i \lambda_{k} t},
$$

the input $u \in B_{a p}^{2}(U)$ given by

$$
u:=-F \tilde{x}+v
$$

is admissible, i.e., $u \in U_{a d}(f)$. Moreover, $\tilde{x}$ is an initially-at-rest solution of (2.1).

Proof. Since $A-B F$ generates an exponentially stable $C_{0}$-semigroup, it immediately follows that $\tilde{x} \in B_{a p}^{2}(H)$ and $u \in B_{a p}^{2}(U)$. A simple calculation shows that the coefficients $\left\{y_{k}\right\}_{k}$ of $B u+f$ are given by

$$
y_{k}=-B F\left(i \lambda_{k} I-A+B F\right)^{-1} z_{k}+z_{k} .
$$

In order to prove the statement it remains to show that

$$
\left(i \lambda_{k} I-A\right)\left(i \lambda_{k} I-A+B F\right)^{-1} z_{k}=-B F\left(i \lambda_{k} I-A+B F\right)^{-1} z_{k}+z_{k} .
$$

This is easy to see, and hence the statement is proved.

REMARK 2.3. If $f$ and $v$ are trigonometric polynomials, then all initially-at-rest solutions corresponding to the input $u=-F \tilde{x}+v$, where $\tilde{x}$ is given by $(2.2)$, are also trigonometric polynomials. Moreover, if $f$ and $v$ are almost periodic functions, then Remark 1.2 implies that $\tilde{x}$, defined by $(2.2)$, also is an almost periodic function. Thus, in this situation the input $u:=-F \tilde{x}+v$ again lies in $A P(U)$.

Now, we are in the position to introduce the optimal control problem. We associate the following cost function with system (2.1)

$J(u, f)=\inf \left\{\|M x(u, f)\|_{2}^{2}+\left\|N^{1 / 2} u\right\|_{2}^{2}: x(u, f) \|_{2}^{2}\right.$ is an initially-at-rest solution of (2.1) $\}$,

where $M \in \mathcal{L}(H), 0<N \in \mathcal{L}(U)$ has a bounded inverse $N^{-1}, f \in B_{a p}^{2}(H)$ and $u \in U_{a d}(f)$. The pair $(M, A)$ is assumed to be detectable. Da Prato and Ichikawa assumed in their paper [9] that every solution of (2.1) is unique. Since we have seen previously that this is only true if $i \mathbb{R} \subset \rho(A)$, we have to minimize over all initiallyat-rest solutions. In the situation of $i \mathbb{R} \subset \rho(A)$, we obtain that the cost function is given by

$$
J(u, f)=\|M x(u, f)\|_{2}^{2}+\left\|N^{1 / 2} u\right\|_{2}^{2},
$$

where $x(u, f)$ is the unique initially-at-rest solution of (2.1).

Our control problem now is, given an $f \in B_{a p}^{2}(H)$, to find an optimal control $u^{o p t} \in U_{a d}(f)$ that minimizes the cost function. The following proposition is well known and can be found in, e.g., [5]. 
Proposition 2.4. There exists a unique $0 \leq Q \in \mathcal{L}(H)$ satisfying the algebraic Riccati equation

$$
\langle A x, Q y\rangle+\langle Q x, A y\rangle+\left\langle\left(M^{*} M-Q B N^{-1} B^{*} Q\right) x, y\right\rangle=0, \quad x, y \in D(A) .
$$

Moreover, $A-B N^{-1} B^{*} Q$ generates an exponentially stable $C_{0}$-semigroup denoted by $T_{-B N^{-1} B^{*} Q}(t)$.

We define the operators $\Phi_{-B N^{-1} B^{*} Q}, \Psi \in \mathcal{L}(T P(H), T P(H))$ by

$$
\left(\Phi_{-B N^{-1} B^{*} Q} f\right)(t):=\int_{-\infty}^{t} T_{-B N^{-1} B^{*} Q}(t-s) f(s) d s
$$

and

$$
(\Psi f)(t):=\int_{t}^{\infty} T_{-B N^{-1} B^{*} Q}^{*}(s-t) Q f(s) d s .
$$

Lemma 1.1 shows that $\Phi_{-B N^{-1} B^{*} Q}$ and $\Psi$ have unique extensions to $B_{a p}^{2}(H)$ (again denoted by $\Phi_{-B N^{-1} B^{*} Q}$ and $\Psi$ ). Moreover, with $f \in T P(H), \Psi f$ is a solution in $T P(H)$ of

$$
\dot{r}(t)+\left(A^{*}-Q B N^{-1} B^{*}\right) r(t)+Q f(t)=0 .
$$

The following proposition proves a useful formula for the cost function.

Proposition 2.5. For every $f \in B_{a p}^{2}(H)$ and every $u \in U_{a d}(f)$ the cost function is given by

$$
\begin{aligned}
J(u, f)= & 2 \operatorname{Re}\langle\Psi f, f\rangle_{2}-\left\|N^{-1 / 2} B^{*} \Psi f\right\|_{2}^{2} \\
& +\inf \left\{\left\|N^{1 / 2}\left(u+N^{-1} B^{*}(Q x(u, f)+\Psi f)\right)\right\|_{2}^{2} \mid x(u, f)\right. \text { is an } \\
& \text { initially-at-rest solution of }(2.1)\} .
\end{aligned}
$$

Proof. (a) First we prove that the statement holds for trigonometric polynomials. Let $u$ and $f$ be trigonometric polynomials such that $u \in U_{a d}(f)$ and choose $x(u, f)$ to be an initially-at-rest solution of (2.1). Note that in this situation $x:=x(u, f)$ is a classical solution of (2.1). Then $u$ and $x:=x(u, f)$ are differentiable and we get (using (2.5) and (2.4))

$$
\begin{aligned}
\frac{d}{d t}[\langle Q x(t), x(t)\rangle+2 \operatorname{Re}\langle(\Psi f)(t), x(t)\rangle]= & -\left\langle x(t), M^{*} M x(t)\right\rangle-\langle N u(t), u(t)\rangle \\
& +\left\|N^{1 / 2}\left(u(t)+N^{-1} B^{*}(Q x(t)+(\Psi f)(t))\right)\right\|^{2} \\
& -\left\langle N^{-1} B^{*}(\Psi f)(t), B^{*}(\Psi f)(t)\right\rangle \\
& +2 \operatorname{Re}\langle(\Psi f)(t), f(t)\rangle .
\end{aligned}
$$

Integrating from $-T$ to $T$, dividing by $2 T$, and letting $T \rightarrow \infty$ we obtain (using the boundedness of $x(-T), x(T),(\Psi f)(-T)$, and $(\Psi f)(T))$

$$
\|M x\|_{2}^{2}+\left\|N^{1 / 2} u\right\|_{2}^{2}=\left\|N^{1 / 2}\left(u+N^{-1} B^{*}(Q x+\Psi f)\right)\right\|_{2}^{2}+2 \operatorname{Re}\langle\Psi f, f\rangle_{2}-\left\|N^{-1 / 2} B^{*} \Psi f\right\|_{2}^{2} .
$$

Thus the formula holds for trigonometric polynomials.

(b) We now prove that the statement holds for arbitrary $f \in B_{a p}^{2}(H)$ and $u \in$ $U_{a d}(f)$. Let the Fourier series of $f$ and $u$ be given by

$$
f(t) \sim \sum_{k=1}^{\infty} f_{k} e^{i \lambda_{k} t} \text { and } u(t) \sim \sum_{k=1}^{\infty} u_{k} e^{i \lambda_{k} t} .
$$


We now define for $K \in \mathbb{N}$

$$
f_{K}(t):=\sum_{k=1}^{K} a_{k} e^{i \lambda_{k} t} \text { and } u_{K}(t):=\sum_{k=1}^{K} u_{k} e^{i \lambda_{k} t} .
$$

Using $f_{K} \rightarrow f$ as $K \rightarrow \infty$ in $B_{a p}^{2}(H)$ and $\Psi \in \mathcal{L}\left(B_{a p}^{2}(H), B_{a p}^{2}(H)\right)$, we obtain

$$
\Psi f_{K} \rightarrow \Psi f \quad \text { as } K \rightarrow \infty \text { in } B_{a p}^{2}(H) .
$$

Choosing an initially-at-rest solution $x(u, f)$ of $(2.1)$ with Fourier series

$$
x(u, f)(t) \sim \sum_{k=1}^{\infty} x_{k} e^{i \lambda_{k} t}
$$

it is easy to see that

$$
x\left(u_{K}, f_{K}\right)(t):=\sum_{k=1}^{K} x_{k} e^{i \lambda_{k} t}
$$

is an initially-at-rest solution of $\dot{x}(t)=A x(t)+B u_{K}(t)+f_{K}(t)$. Moreover, in part (a), we have showed

$$
\begin{aligned}
\left\|M x_{K}(u, f)\right\|_{2}^{2}+\left\|N^{1 / 2} u_{K}\right\|_{2}^{2}= & \left\|N^{1 / 2}\left(u_{K}+N^{-1} B^{*}\left(Q x\left(u_{K}, f_{K}\right)+\Psi f_{K}\right)\right)\right\|_{2}^{2} \\
& +2 \operatorname{Re}\left\langle\Psi f_{K}, f_{K}\right\rangle_{2}-\left\|N^{-1 / 2} B^{*} \Psi f_{K}\right\|_{2}^{2} .
\end{aligned}
$$

Since $u_{K} \rightarrow u$ and $x\left(u_{K}, f_{K}\right) \rightarrow x(u, f)$ as $K \rightarrow \infty$ in $B_{a p}^{2}(H)$ and $B_{a p}^{2}(U)$, respectively, we get immediately from (2.3)

$$
\left\|M x\left(u_{K}, f_{K}\right)\right\|_{2}^{2}+\left\|N^{1 / 2} u_{K}\right\|_{2}^{2} \rightarrow\|M x(u, f)\|_{2}^{2}+\left\|N^{1 / 2} u\right\|_{2}^{2} \text { as } K \rightarrow \infty .
$$

Moreover, the right-hand side of (2.7) converges for $K \rightarrow \infty$ to

$$
\left\|N^{1 / 2}\left(u+N^{-1} B^{*}(Q x(u, f)+\Psi f)\right)\right\|_{a p}^{2}+2 \operatorname{Re}\langle\Psi f, f\rangle_{2}-\left\|N^{-1 / 2} B^{*} \Psi f\right\|_{2}^{2} .
$$

Thus

$$
\begin{aligned}
\|M x(u, f)\|_{a p}^{2}+\left\|N^{1 / 2} u\right\|_{2}^{2}= & \left\|N^{1 / 2}\left(u+N^{-1} B^{*}(Q x(u, f)+\Psi f)\right)\right\|_{2}^{2} \\
& +2 \operatorname{Re}\langle\Psi f, f\rangle_{2}-\left\|N^{-1 / 2} B^{*} \Psi f\right\|_{2}^{2},
\end{aligned}
$$

which completes the proof.

The solution of the control problem is given by the following theorem.

ThEOREM 2.6. For every $f \in B_{a p}^{2}(H)$ there exists a unique optimal control $u^{o p t} \in U_{a d}(f)$ that minimizes the cost function. This optimal control is given by

$$
u^{o p t}=-N^{-1} B^{*}(Q \tilde{x}+\Psi f),
$$

where $\tilde{x}=\Phi_{-B N^{-1} B^{*} Q}\left[-B N^{-1} B^{*} \Psi f+f\right]$, and the minimal cost is given by

$$
J\left(u^{o p t}, f\right)=2 \operatorname{Re}\langle\Psi f, f\rangle_{2}-\left\|N^{-1 / 2} B^{*} \Psi f\right\|_{2}^{2} .
$$

Proof. Proposition 2.4 shows that $A-B N^{-1} B^{*} Q$ generates an exponentially stable $C_{0}$-semigroup. Therefore, $\Psi f$ and $\tilde{x}$ are elements of $B_{a p}^{2}(H)$. Proposition 2.2 implies that $u^{o p t}$ as defined in (2.8) is an element of $U_{a d}(f)$ and that $\tilde{x}$ is an initiallyat-rest solution of $\dot{x}(t)=A x(t)+B u(t)+f(t)$ and $\tilde{x}=x\left(u^{o p t}, f\right)$. Now equation (2.6) shows that the admissible control $u^{\text {opt }}$ minimizes the cost function, and that 
the optimal cost is given by (2.9). It now remains to show that $u^{o p t}$ is the unique optimal control. Let $u \in U_{a d}(f)$ be chosen in such a way that $u$ minimizes the cost function and let $x(u, f)$ be the corresponding minimizing initially-at-rest solution of (2.1). Moreover, let the Fourier series of $u, x(u, f), f$, and $\Psi f$ be given by

$$
\begin{aligned}
u(t) & \sim \sum_{k=1}^{\infty} u_{k} e^{i \lambda_{k} t}, & & x(u, f)(t) \sim \sum_{k=1}^{\infty} x_{k} e^{i \lambda_{k} t}, \\
f(t) & \sim \sum_{k=1}^{\infty} f_{k} e^{i \lambda_{k} t}, & & (\Psi f)(t) \sim \sum_{k=1}^{\infty} p_{k} e^{i \lambda_{k} t} .
\end{aligned}
$$

By the definition of an initially-at-rest solution and by the fact that $u$ minimizes the cost function we have

1. $\left(i \lambda_{k} I-A\right) x_{k}=B u_{k}+f_{k}$ for every $k \in \mathbb{N}$,

2. $u_{k}+N^{-1} B^{*} Q x_{k}+N^{-1} B^{*} p_{k}=0$ for every $k \in \mathbb{N}$.

Thus $x_{k}$ has to be

$$
x_{k}=\left(i \lambda_{k} I-A+B N^{-1} B^{*} Q\right)^{-1}\left[-B N^{-1} B^{*} p_{k}+f_{k}\right] .
$$

This implies immediately that $x(u, f)$ is given by

$$
x(u, f)=\Phi_{-B N^{-1} B^{*} Q}\left[-B N^{-1} B^{*} \Psi f+f\right]
$$

and that $u$ is given by $u=-N^{-1} B^{*}(Q x(u, f)+\Psi f)$, which completes the proof.

If $f \in T P(H)$, then the optimal control $u^{o p t}$ is also a trigonometric polynomial. Moreover, $u^{o p t}$ is an almost periodic function if $f \in A P(H)$ (see Remark 2.3).

Acknowledgment. The authors would like to thank Ruth Curtain for bringing them together to work on this problem. We also would like to thank an anonymous referee for pointing out some useful references.

\section{REFERENCES}

[1] A. Avantaggiati, G. Bruno, and R. Iannacci, A functional approach to $B_{q}$-a.p. spaces and $L^{\infty}$ Fourier expansions, Rend. Mat. Appl. VII, 13 (1993), pp. 199-228.

[2] A. S. Besicovitch, Almost Periodic Functions, Cambridge University Press, London, 1932.

[3] H. Bohr, Almost Periodic Functions, Chelsea, New York, 1947.

[4] J. M. Borwein And P. B. Borwein, Pi and the AGM, Wiley-Interscience, New York, 1987.

[5] R. F. Curtain and H. J. Zwart, An Introduction to Infinite-Dimensional Linear Systems Theory, Springer-Verlag, New York, 1995.

[6] C. Corduneanu, Almost Periodic Functions, Wiley-Interscience, New York, 1968.

[7] H. Kwakernaak and R. Sivan, Modern Signals and Systems, Prentice Hall, Englewood Cliffs, NJ, 1991.

[8] M. Larsen, $H_{\infty}$ Tracking Problems for a Distributed Parameter System, Ph.D. thesis, Mathematical Institute, Technical University of Denmark, Lyngby, 1997.

[9] G. Da Prato And A. IChIKaWa, Optimal control of linear systems with almost periodic inputs, SIAM J. Control Optim., 25 (1987), pp. 1007-1019.

[10] A. C. ZAAnen, Linear Analysis, North-Holland, Amsterdam, 1960. 